\title{
Treatment Cost for Typhoid Fever at Two Hospitals in Kolkata, India
}

\author{
Dipika Sur', Susmita Chatterjee',Arthorn Riewpaiboon², Byomkesh Manna', \\ Suman Kanungo', and Sujit K. Bhattacharya ${ }^{3}$ \\ 'National Institute of Cholera and Enteric Diseases, Kolkata, India, ${ }^{2}$ Faculty of Pharmacy, Division of Social and Administrative \\ Pharmacy, Mahidol University, Bangkok, Thailand, and ${ }^{3}$ Indian Council of Medical Research, New Delhi, India
}

\begin{abstract}
The purpose of this study was to estimate treatment cost for typhoid fever at two hospitals in Kolkata, India. This study was an incidence-based cost-of-illness analysis from the providers' perspective. Microcosting approach was employed for calculating patient-specific data. Unit costs of medical services used in the calculation were directly measured from the study hospital by standard method. The study hospitals were selected based on accessibility to data and cooperation. Eighty-three Widal-positive and/or cultureconfirmed patients with typhoid fever during November 2003-April 2006 were included in the study. Most (93\%) patients were children. Eighty-one percent was treated at the outpatient department. The average duration of hospitalization for child and adult patients was 8.4 and 4.2 days respectively. The average cost of treating children, adults, and all patients was US\$ 16.72, 72.71, and 20.77 respectively (in 2004 prices). Recalculation based on $80 \%$ occupancy rate in inpatient wards (following the recommendation of the World Health Organization) found that the cost of treating children, adults, and all patients was US\$14.53, 36.44 , and 16.11 respectively.
\end{abstract}

Key words: Costs of illness; Healthcare cost; Treatment cost; Typhoid fever; India

\section{INTRODUCTION}

Typhoid fever has been an important health problem in many parts of the world. It is estimated that 16 million cases and 600,000 deaths occur each year (1). The typhoid vaccines have been introduced in many countries including India (2). In India, the cost of illness from typhoid fever and vaccine-evaluation studies were also conducted in 1996 in New Delhi $(3,4)$. However, India is a vast country, and the states are very big and are quite different in environment and health service patterns. Hence, results of studies in one state may not be sufficient to be generalized for different states and for the whole country. The present study was an attempt to estimate the cost of illness from typhoid fever at two hospitals in Kolkata.

Kolkata, a district of the state of West Bengal, has

Correspondence and reprint requests should be

addressed to:

Dr. Susmita Chatterjee

Economist

National Institute of Cholera and Enteric Diseases

P 33, CIT Road, Scheme XM

Beliaghata, Kolkata 700010

India

Telephone: 919830834280

Email: s_chatterjee_123@yahoo.com an area of approximately $185 \mathrm{sq} \mathrm{km}$ and had a total population of 4,572,876 in 2001 (Census of India 2001). The urban area has an estimated populationdensity of 24,718 persons per sq $\mathrm{km}$. About $32.5 \%$ of the total population in Kolkata lives in slum areas (Census of India 2001). Typhoid fever, characterized by high fever, chills, headache, and malaise or delirium, is one of the health problems in West Bengal, especially in the overcrowded slums in Kolkata. A surveillance based on two urban slums of Kolkata conducted during November 2003-October 2004 found that the incidence of typhoid fever per 1,000 per year was 0.9 for children aged less than two years, 3.4 for the age-group of 2-4.99 years, 4.9 for the age-group of 5-14.99 years, and 1.2 for the age group of 15 years and above (5). Lack of proper sanitation, safe drinking-water, hygienic practices, and health education results in high prevalence of this disease among slum dwellers. Although improvements in water and sanitation infrastructure and food-hygiene could reduce the burden of disease and lessen the threat of antibiotic resistance, another strategy to reduce typhoid cases in the near term is vaccination with new-generation vaccines (6). To introduce a vaccine to the health service system, the burden of disease, including economic consequences, must be explored to determine priority. In addition, information on economic burden 
or cost of illness is important in the evaluation of vaccination programmes. Costs of illness can be estimated from private and public points of view. Private cost of illness focuses on the treatment cost borne by the patients themselves or the caregivers (in the case of child patients) while public cost of illness estimates the total cost incurred by a healthcare provider to treat a particular patient, i.e. the public cost-of-illness study provides estimates from the perspective of the healthcare system. The present study was a public cost-of-illness study aimed at estimating treatment cost for typhoid fever at two hospitals in Kolkata, viz. the B C Roy Memorial Hospital for Children and the Infectious Diseases \& Beliaghata General Hospital.

\section{MATERIALS AND METHODS}

Cost-of-illness studies use either a prevalencebased or incidence-based approach, depending on whether a fixed-time horizon or life-time horizon is adopted as the conceptual paradigm (7). The prevalence-based cost-of-illness studies measure the economic burden of a disease in a given period. In contrast, the incidence-based approach measures the economic burden from the onset of disease until cure or death. It observes only new cases occurring in a given period and monitors until the end-point is reached. Hence, in the present study, the incidence-based approach was used. This study was analyzed from the provider's perspective (7).

To select the study site, retrospective surveys on hospital data were carried out during 2002-2003 in two hospitals in Kolkata-the Infectious Diseases \& Beliaghata General Hospital and the B C Roy Memorial Hospital for Children. These two hospitals were selected based on accessibility to data and cooperation. The survey results showed that most patients with enteric diseases admitted or treated as outpatients at these two hospitals were from two Kolkata municipal wards, viz. Ward 29 and 30. Hence, these two wards were selected for the present study. Ward 29 and 30 are very congested urban slums capturing an area of about $0.7 \mathrm{sq} \mathrm{km}$ and have a total population of 75,165.

The B C Roy Memorial Hospital for Children is a teaching hospital specializing in paediatric medicine; it has approximately 250 beds, 51 doctors, 90 nurses, 20 supporting staff (pathologist, physiotherapist, etc.), 26 administrative staff, and 125 Group D staff (sweeper, peon, etc.). The hospital provides both inpatient and outpatient care. On an average, 500 patients are treated as outpatients daily. The Infectious Diseases \& Beliaghata General Hospital has 560 beds. Most common infectious diseases treated in this hospital are diarrhoea, diph- theria, whooping cough, tetanus (including neonatal tetanus), rabies, encephalitis, complicated cases of measles and chicken pox, and a few other infectious diseases. The hospital also has an outpatients department where approximately 150 patients are seen daily. In total, 62 doctors take care of these patients with the help of 157 nurses, 21 paramedical staff, and 252 Group D staff.

Treating patients with typhoid fever is a part of the hospital services. Based on routine practice, patients are clinically diagnosed and treated by the hospital doctors. However, for some patients, laboratory investigations are performed for confirmation. For example, Widal test is often performed by the hospital for patients with typhoid fever. All typhoid-positive cases (Widal-positive, i.e. TO (titre of antibody developed against ' $\mathrm{O}$ ' antigen of typhoid causing bacteria) $\geq 80$ or culture-confirmed) admitted or treated as outpatients at the B C Roy Memorial Hospital for Children and at the Infectious Diseases \& Beliaghata General Hospital from Ward 29 and 30 during November 2003-April 2006 were included in the present study. In total, 83 cases were included. These patients were identified from the ongoing passive surveillance of the Diseases of Most Impoverished (DOMI) programme in these two wards. The DOMI programme, administered by the International Vaccine Institute, Seoul, South Korea, involved a number of parallel activities, such as epidemiological studies, economic studies, and investigation of the feasibility of vaccine-technology transfer in several Asian countries, including India. Surveillance was a major part of the whole study, and results of the surveillance showed that, during the present study period, the total number of typhoid-positive cases was 2,658, of which 2,285 were widal-positive cases $(\mathrm{TO} \geq 80)$ and 373 were culture-confirmed cases. As part of the DOMI programme, five health outposts were set up in ward 29 and 30 and one each at the Infectious Diseases \& Beliaghata General Hospital and B C Roy Memorial Hospital for Children. In addition to passive surveillance, the community health workers visited each household in the study areas on a monthly basis to encourage febrile patients to attend those participating healthcare facilities. The private practitioners around the study areas were also encouraged to refer patients for diagnosis and treatment free of charge at those facilities (5). As a result, patients from the study area with symptoms of typhoid fever were diagnosed and treated at the local healthcare facilities at a very early stage of the disease, and the disease did not become severe. This was evident from the results of the surveillance which showed that only $2 \%$ of typhoid cases required hospitaliza- 
tion, and all detected cases were recovered, and no death due to typhoid was reported during November 2003-October 2004 (5). Clearly, because of this intervention, only a few patients were admitted or treated as outpatients at the study hospitals, although there were substantial numbers of typhoidpositive cases.

For this study, resources used by the hospitals and service outputs were collected from the official records of the two hospitals. The hospital-related data were collected for the 2004-2005 financial year. Hence, the cost calculation of this study was based on the 2004 prices. The clinical status and the treatment provided to each patient were collected from the ongoing surveillance programme in the two wards.

Cost-of-illness is composed of direct medical, nonmedical, and indirect costs (7). However, as results of the present study were analyzed from the provider's perspective, it covered only the provider's cost, i.e. direct medical cost. Direct medical cost is healthcare-related costs directly spent for the prevention, detection, treatment, continuing care, rehabilitation, and terminal care of patients (7). Direct medical cost was calculated by multiplying the quantity of medical services consumed by their unit costs. A micro-costing bottom-up approach was used for calculating the unit cost of medical services (Fig. 1) (8). The calculation was composed of five steps: organization analysis and cost centre classification, determination of direct cost, determination of indirect cost, determination of full cost, and calculating unit cost of medical services (Fig. 2).

At first, all departments of the hospital were classified into patient-care cost centres and supporting cost centres. The direct cost of each cost centre was calculated by summing up its labour, material and capital costs. Labour cost was the summation of salaries, overtime pay, and other fringe benefits, such as healthcare expenses, travel allowance, etc. Material costs included costs of maintenance, electricity, telephone, petrol, meals, security, and miscellaneous expenses. For limitation to access data of capital assets, capital cost was estimated at 15\% of the total cost. This was an approximate average proportion from reports in Thailand: $17.8 \%$ in a 60-bed hospital (9), 15.89\% in a 509-bed hospital (10), 11\% in a 30-bed hospital (11).

After deriving the direct cost of all the cost centres, the direct costs of the supporting cost centres were allocated to patient-care cost centres by employing simultaneous equation method (12). The direct costs of the supporting cost centres, after being allo- cated to the patient-care cost centres, were termed as indirect cost of the patient-care cost centres. Full cost of each patient-care cost centre was obtained by adding up direct and indirect costs.

For the calculation of unit cost, the departments producing homogeneous products, such as pharmacy, outpatient department, and inpatient department, average method was used $(13,14)$. For example, to get the unit cost per outpatient visit, full cost of the outpatient department was divided by the total number of outpatient visits at the hospital during the whole year. However, to calculate the unit cost of services produced at the pathology department (heterogeneous output), ratio of costs to charges (RCC) method was used $(13,14)$. RCC is computed when the information on the full cost of the department, prices, and total number of services produced is available. In this calculation process, total expected charge was calculated first by multiplying unit prices of services and total number of services produced. Then, full cost of the department was divided by total expected charge resulting in the ratio of cost to charge. Finally, that ratio was used for multiplying unit price, resulting in unit cost of each service.

The final step was to calculate treatment cost of an individual patient. As part of the surveillance programme of the DOMI project, a clinical record form was designed to record service-use by each patient. Drugs and their quantities used by each patient were drawn from the clinical record form and then multiplied by drug-prices to retailer from the Drug Today (15), resulting in acquisition drug cost. Dispensing cost was added to acquisition drug cost, resulting in gross pharmacy cost. The numbers of outpatient visits, hospitalization days, and laboratory tests were multiplied by their unit costs. Total treatment cost was obtained by adding up all these costs. The present study was based on the assumption of real-life practice. Hence, the laboratory tests, e.g. Widal test or culture done for the confirmation of the pathogen for the research purpose, were not included in the calculation of cost. This was consistent with the exclusion criteria mentioned in the guidelines for economic evaluations in pharmaceuticals (16). However, costs of laboratory tests that were parts of treatment were included. All costs were presented in US dollar at the 2004 prices.

Hence, types of cost for this study were the following:

a. Laboratory cost consisting of practical laboratory investigations-the test used for research purpose-was not included. 


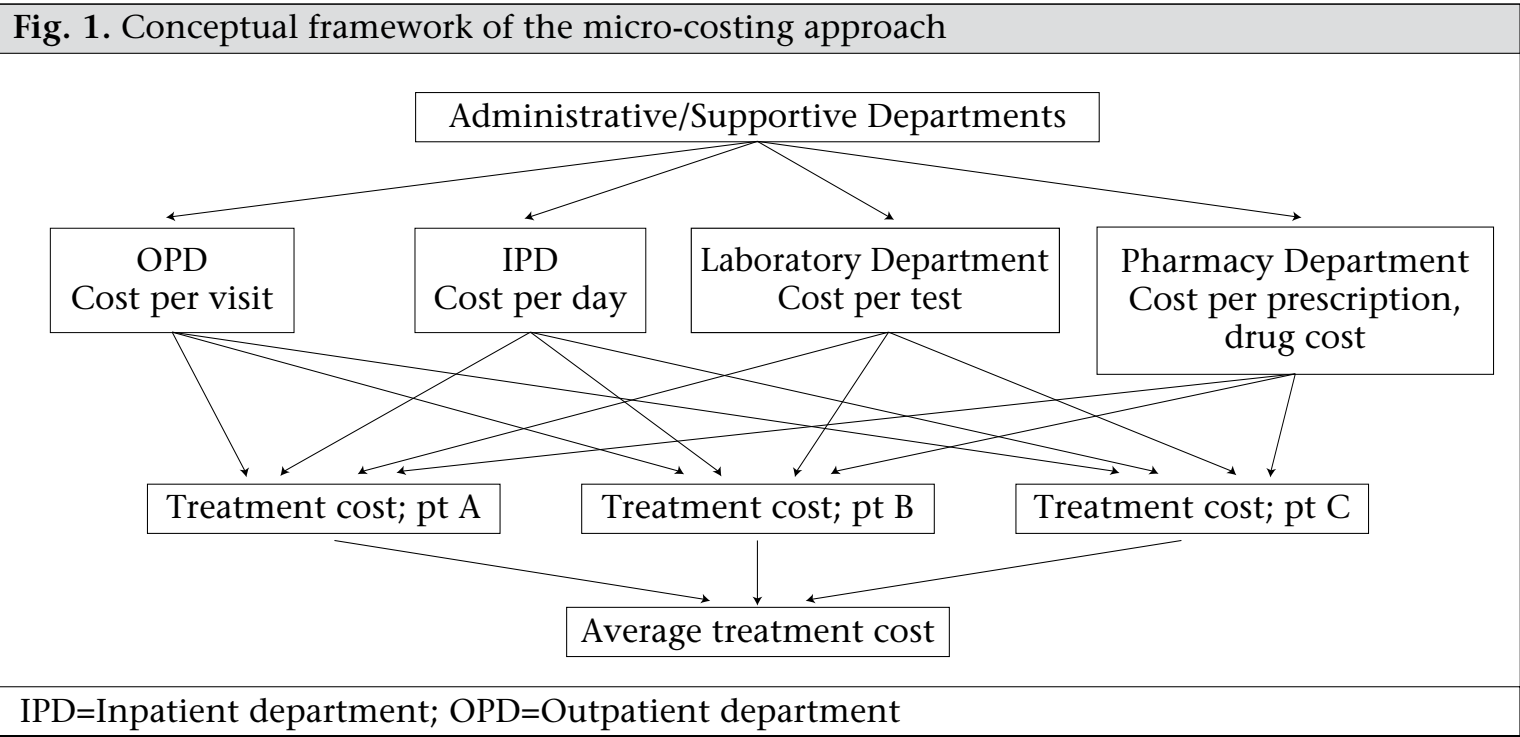

b. Pharmacy cost equalled acquisition drug cost plus drug-dispensing cost

c. Routine service cost was the sum of outpatient and inpatient service.

d. Total cost or total treatment cost consisting of all costs mentioned above (points $\mathrm{a}, \mathrm{b}$, and $\mathrm{c}$ ).

\section{RESULTS}

The results were presented as outpatient and inpatient treatment costs. The patients were classified as children (aged less than 18 years) and adults (aged 18 years or more). Descriptive statistics were employed in the analysis. Based on available data, the Infectious Diseases \& Beliaghata General Hospital and the B C Roy Memorial Hospital for Children had $34 \%$ and $75 \%$ occupancy rates of hospitalization services respectively. Following the recommendation of the World Health Organization, sensitivity analysis was conducted at $80 \%$ of capacity-use (17).

Unit cost of medical services was calculated for estimating treatment cost. Two study hospitals had different levels of capacity-use. Inpatient-bed occupancy rate of the B C Roy Memorial Hospital for Children was two times higher than that of the

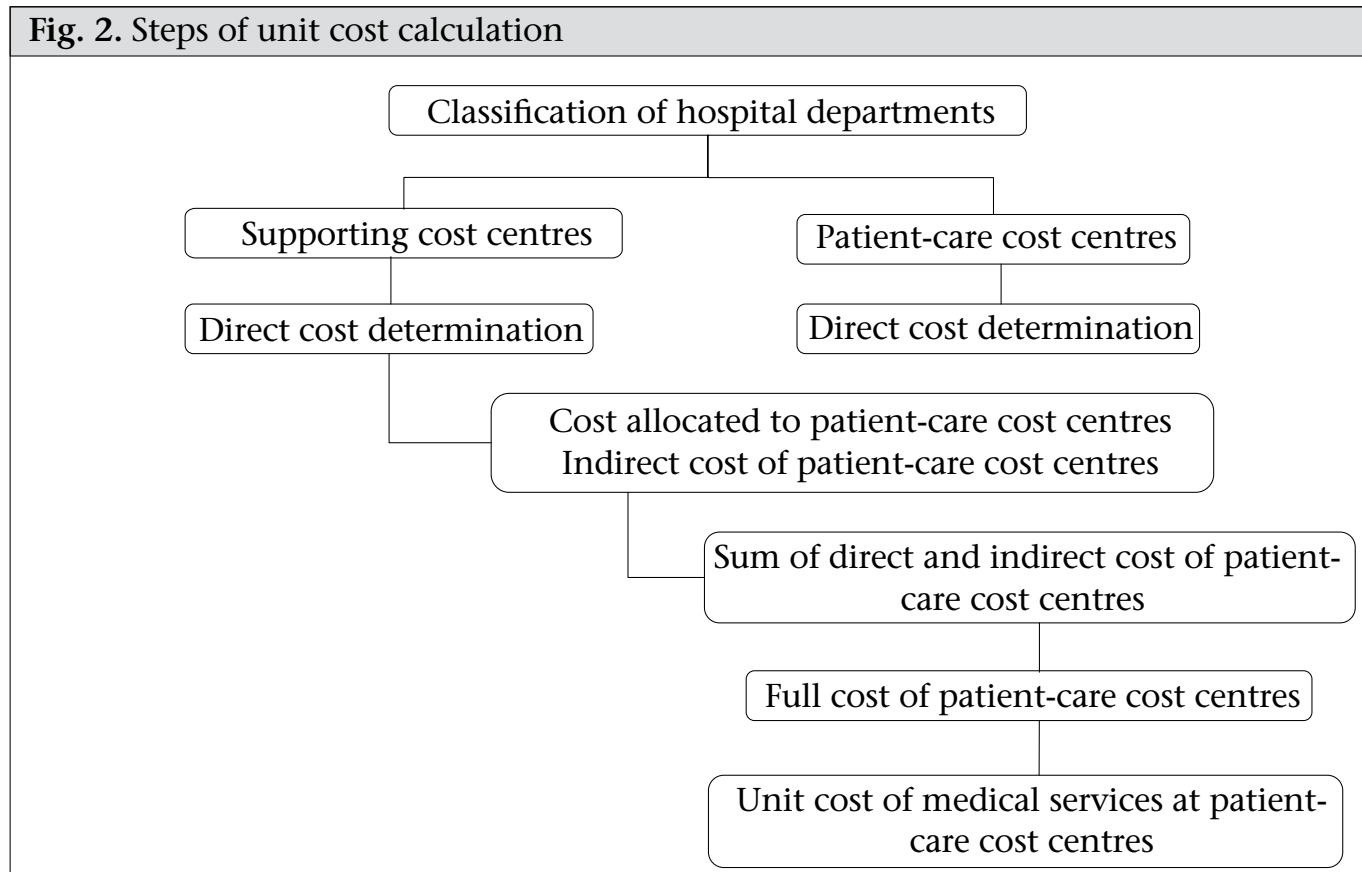




\begin{tabular}{|c|c|c|}
\hline Output & $\begin{array}{l}\text { Infectious Diseases \& Belia- } \\
\text { ghata General Hospital }\end{array}$ & $\begin{array}{l}\text { B C Roy Memorial Hospital for } \\
\text { Children }\end{array}$ \\
\hline Number of beds & 560 & 250 \\
\hline Admission/day & 67 & 24 \\
\hline Occupancy rate & $34 \%$ & $75 \%$ \\
\hline Average length (days) of stay & 2.89 & 7.98 \\
\hline Total hospital cost (acquisition) & $1,356,843$ & $1,335,569$ \\
\hline Cost per outpatient visit (US\$*) & 0.37 & 0.69 \\
\hline Cost per inpatient day $\left(\mathrm{US}^{*}{ }^{*}\right)$ & 15.27 & 10.80 \\
\hline
\end{tabular}

Infectious Diseases \& Beliaghata General Hospital (75\% and 34\% respectively). Costs per inpatient day were US\$ 10.80 and US\$ 15.27 for the B C Roy Memorial Hospital for Children and the Infectious Diseases \& Beliaghata General Hospital respectively. In contrast, outpatient routine service (or consultation) costs were US $\$ 0.69$ and US\$ 0.37 for the B C Roy Memorial Hospital for Children and the Infectious Diseases \& Beliaghata General Hospital respectively (Table 1).
Most included patients were children (77 of 83 cases, 93\%) and outpatients (81\%). There were no adult outpatients. The average age was 6.16 years and 22.67 years for children and adults respectively. The average length of stay at the hospital was 6.81 days. Child inpatients stayed longer than adult patients (8.40 vs 4.17 days). Details are presented in Table 2.

Table 3 shows composition of treatment cost. It was found that pharmacy cost (acquisition drug cost and dispensing-service cost) was approximate-

\begin{tabular}{|c|c|c|c|c|c|c|}
\hline \multirow{2}{*}{ Age-group } & \multirow{2}{*}{ No. } & \multirow{2}{*}{$\%$} & \multicolumn{2}{|c|}{ Age (years) } & \multicolumn{2}{|c|}{ Length (days) of stay } \\
\hline & & & Mean & $\mathrm{SD}$ & Mean & SD \\
\hline \multicolumn{7}{|l|}{ Children } \\
\hline Hospitalized & 10 & 13.0 & 7.90 & 4.01 & 8.40 & 2.07 \\
\hline Outpatients & 67 & 87.0 & 5.90 & 3.02 & NA & NA \\
\hline Both types & 77 & 100.0 & 6.16 & 3.21 & NA & NA \\
\hline \multicolumn{7}{|l|}{ Adults } \\
\hline Hospitalized & 6 & 100.0 & 22.67 & 6.28 & 4.17 & 2.93 \\
\hline Outpatients & 0 & 0.0 & NA & NA & NA & NA \\
\hline Both types & 6 & 100.0 & 22.67 & 6.28 & NA & NA \\
\hline \multicolumn{7}{|l|}{ All ages } \\
\hline Hospitalized & 16 & 19.3 & 13.44 & 8.79 & 6.81 & 3.15 \\
\hline Outpatients & 67 & 80.7 & 5.90 & 3.02 & NA & NA \\
\hline Both types & 83 & 100.0 & 7.35 & 5.52 & NA & NA \\
\hline
\end{tabular}

Table 3. Total cost (US\$) of study sample ( $\mathrm{n}=83$ ) at 2004 prices*

\begin{tabular}{|lccccc|}
\hline \multirow{2}{*}{ Category } & \multicolumn{2}{c}{ Base case } & & \multicolumn{2}{c|}{$80 \%$ occupancy rate } \\
\cline { 2 - 3 } & $\begin{array}{c}\text { Mean } \\
\text { cost }\end{array}$ & $\%$ & & $\begin{array}{c}\text { Mean } \\
\text { cost }\end{array}$ & $\%$ of change \\
\hline Laboratory cost & 83.33 & 4.83 & & 83.33 & - \\
Pharmacy cost & 242.64 & 14.08 & & 242.64 & - \\
Routine service cost & $1,397.67$ & 81.09 & & $1,011.27$ & 27.65 \\
Total treatment cost & $1,723.64$ & 100 & & $1,337.24$ & 22.42 \\
\hline${ }^{*}$ US\$ 1=Indian Rs 44 & & & & \\
\hline
\end{tabular}


ly $15 \%$ of the treatment cost. The major part of the cost (approximately 80\%) was routine-service cost, particularly that for the hospitalization. It was also found that if the number of inpatient days increased to result in $80 \%$ occupancy rate, the cost decreased by nearly one-fourth.
The average treatment costs are presented in Table 4 . For base case, the average treatment cost of typhoid patients was US\$20.77. However, there was a substantial difference between outpatient and inpatient costs (US\$ 2 vs US\$ 99.36). The average cost of hospitalized child patient was US\$ 115.36. This

\begin{tabular}{|c|c|c|c|c|}
\hline \multirow{2}{*}{ Age-group } & \multicolumn{2}{|c|}{ Base case } & \multicolumn{2}{|c|}{$80 \%$ occupancy rate } \\
\hline & Mean & SD & Mean & SD \\
\hline \multicolumn{5}{|l|}{ Children } \\
\hline \multicolumn{5}{|c|}{ Hospitalized $(\mathrm{n}=10,13 \%)$} \\
\hline Laboratory & 8.33 & 17.45 & 8.33 & 17.45 \\
\hline Pharmacy & 10.08 & 11.31 & 10.08 & 11.31 \\
\hline Routine service & 96.94 & 22.07 & 80.06 & 26.30 \\
\hline Total & 115.36 & 28.67 & 98.48 & 34.25 \\
\hline \multicolumn{5}{|c|}{ Outpatients (n=67, 87\%) } \\
\hline Laboratory & $0^{*}$ & NA & $0^{*}$ & NA \\
\hline Pharmacy & 1.30 & 1.11 & 1.30 & 1.11 \\
\hline Routine service & $0.69^{*}$ & NA & $0.69^{*}$ & NA \\
\hline Total & 2.00 & 1.11 & 2.00 & 1.11 \\
\hline \multicolumn{5}{|c|}{ Both types $(n=77,100 \%)$} \\
\hline Laboratory & 1.08 & 6.63 & 1.08 & 6.63 \\
\hline Pharmacy & 2.44 & 5.00 & 2.44 & 5.00 \\
\hline Routine service & 13.19 & 33.44 & 11.00 & 28.34 \\
\hline Total & 16.72 & 39.62 & 14.53 & 34.72 \\
\hline \multicolumn{5}{|l|}{ Adults } \\
\hline \multicolumn{5}{|c|}{ Hospitalized $(6,100 \%)$} \\
\hline Laboratory & $0^{*}$ & NA & $0^{*}$ & NA \\
\hline Pharmacy & 9.09 & 9.72 & 9.09 & 9.72 \\
\hline Routine service & 63.62 & 44.69 & 27.35 & 19.21 \\
\hline Total & 72.71 & 53.05 & 36.44 & 27.83 \\
\hline \multicolumn{5}{|l|}{ All ages } \\
\hline \multicolumn{5}{|c|}{ Hospitalized $(\mathrm{n}=16,19.3 \%)$} \\
\hline Laboratory & 5.21 & 14.14 & 5.21 & 14.14 \\
\hline Pharmacy & 9.71 & 10.42 & 9.71 & 10.42 \\
\hline Routine service & 84.44 & 35.15 & 60.30 & 35.11 \\
\hline Total & 99.36 & 43.43 & 75.22 & 43.87 \\
\hline \multicolumn{5}{|c|}{ Outpatients ( $n=67,80.7 \%)$} \\
\hline Laboratory & $0^{*}$ & NA & $0^{*}$ & NA \\
\hline Pharmacy & 1.30 & 1.11 & 1.30 & 1.11 \\
\hline Routine service & $0.69^{*}$ & NA & $0.69^{*}$ & NA \\
\hline Total & 2.00 & 1.11 & 2.00 & 1.11 \\
\hline \multicolumn{5}{|c|}{ Both types $(n=83,100 \%)$} \\
\hline Laboratory & 1.00 & 6.39 & 1.00 & 6.39 \\
\hline Pharmacy & 2.92 & 5.65 & 2.92 & 5.65 \\
\hline Routine service & 16.83 & 36.48 & 12.18 & 28.02 \\
\hline Total & 20.77 & 42.89 & 16.11 & 34.60 \\
\hline
\end{tabular}

${ }^{*}$ Constant; $\dagger$ Routine service cost consisting of cost of outpatient service (consultation cost) and inpatient service (hotel cost); ‡Routine service cost of outpatient was equal (or constant) for all outpatients. In addition, all outpatients did not have laboratory cost; $\mathrm{NA}=\mathrm{Not}$ applicable; $\mathrm{SD}=\mathrm{Standard}$ deviation 
was much higher than that of the adult patients (US\$ 72.71). These treatment costs were presented for both base case and simulated scenario at $80 \%$ inpatient bed-occupancy rate.

\section{DISCUSSION}

Treatment cost for this study was obtained by multiplying the unit costs of medical services and quantity of the services used. The two hospitals included in this study were quite different in terms of capacity-use, and this was illustrated by a huge difference in the occupancy rates. Hence, treatment costs based on actual situation (base case) might have limitation for generalization. To overcome this limitation, re-analysis of the treatmentcost by employing $80 \%$ of capacity-use in terms of occupancy rate (following the recommendation of WHO) was done (17). Hence, the results obtained by using $80 \%$ occupancy rate should be used for planning at the national level.

While comparing the treatment cost between agegroups, it was found that the hospitalized child patients had higher cost than that of the hospitalized adult patients. This was because the length of stay of child patients was double compared to adults. As routine service cost (at outpatient and inpatient departments) contributed $81 \%$ of the total cost, a longer duration of stay of the child patients led to higher treatment cost for them compared to the adults.

A study in the urban slums of Delhi in 1996 found that the average direct cost of blood culture-confirmed typhoid, blood culture-confirmed paratyphoid, and clinical typhoid was Rs 277 , Rs 74, and Rs 432 respectively (3). These costs were converted into US dollar in 2004 rate, and the same stood at US\$ $12.14,7.63$, and 18.94 respectively (18). The present study also calculated the average treatment cost of blood culture-confirmed typhoid, blood culture-confirmed paratyphoid, and Widal test-positive cases. However, the sample size of the subgroups was too small to present the means separately. Hence, the average treatment cost was reported, and it was US\$ 16.11 , which was quite similar to the average treatment cost found in the study in Delhi.

The present study has some limitations. First, real prices of drugs purchased by the hospital or government pharmaceutical agency were not available. Drug-prices quoted from Drug Today were the quoted prices of pharmaceutical companies. We had no information of whether those prices were higher or lower than those purchased by the hospital. Second, information on capital assets, e.g. equipment, were not available for calculation of the capital cost. The estimation of $15 \%$ of the total cost for capital cost was based on Thailand experience. Furthermore, there were no adult outpatients in this study, and the sample size for adult hospitalized cases was also very small. To estimate more accurate average treatment cost for typhoid, weighted average should be applied. This was not possible in this study due to paucity of data on estimated treatment cost of adult outpatients and proportion of patients (child vs adult) receiving treatment at outpatient and inpatient departments.

\section{ACKNOWLEDGEMENTS}

This research was a part of the Diseases of the Most Impoverished (DOMI) programme, administered by the International Vaccine Institute, Seoul, South Korea, with the support from the Bill \& Melinda Gates Foundation. The objective of the DOMI programme was to accelerate the development and introduction of vaccines against typhoid fever, cholera, and shigellosis. This study was a part of social science studies, the results of which will support public decision-making regarding immunization programme for typhoid fever. The authors are indebted to all staff of the study hospitals for their continuous support at the time of data collection and clarification.

\section{REFERENCES}

1. Hessel L, Debois H, Fletcher M, Dumas R. Experience with Salmonella typhi Vi capsular polysaccharide vaccine. Eur J Clin Microbiol Infect Dis 1999;18:609-20.

2. Acosta CJ, Galindo CM, Ochiai RL, Danovaro-Holliday MC, Page AL, Thiem VD et al. The role of epidemiology in the introduction of Vi polysaccharide typhoid fever vaccines in Asia. J Health Popul Nutr 2004;22:240-5.

3. Bahl R, Sinha A, Poulos C, Whittington D, Sazawal S, Kumar R et al. Costs of illness due to typhoid fever in an Indian urban slum community: implications for vaccination policy. J Health Popul Nutr 2004;22:304-10.

4. Poulos C, Bahl R, Whittington D, Bhan MK, Clemens JD, Acosta CJ. A cost-benefit analysis of typhoid fever immunization programmes in an Indian urban slum community. J Health Popul Nutr 2004;22:311-21.

5. Ochiai RL, Acosta CJ, Danovaro-Holliday MC, Bhattacharya SK, Agtini M et al. A study of typhoid fever in five Asian countries: disease burden and implications for control. Bull World Health Organ 2008;86:260-8. 
6. Cook J, Jeuland M, Whittington D, Poulos C, Clemens J, Sur D et al. The cost-effectiveness of typhoid Vi vaccination programs: calculations for four urban sites in four Asian countries. Vaccine 2008;26:6305-16.

7. Pagano E, Brunetti M, Tediosi F, Garattini L. Costs of diabetes: a methodological analysis of the literature. Pharmacoeconomics 1999;15:583-95.

8. Brouwer W, Rutten F, Koopmanschap M. Costing in economic evaluations. In: Drummond M, McGuire A, editors. Economic evaluation in health caremerging theory with practice. New York, NY: Oxford University Press, 2001:68-93.

9. Riewpaiboon A, Malaroje S, Kongsawatt S. Effect of costing methods on unit cost of hospital medical services. Trop Med Int Health 2007;12:554-63.

10. Riewpaiboon A, Jaroenkitpan N, Wipaswacharayotin Y. Cost structure of hospital-based pharmaceutical services: a consideration of reimbursement. Mahidol Univ J Pharm Sci 2005;32:47-54.

11. Riewpaiboon A, Pornlertwadee P, Pongsawat K. Diabetes cost model of a hospital in Thailand. Value in Health 2007;10:223-30.
12. Drummond MF, Sculpher MJ, Torrance GW, O'Brien BJ, Stoddart GL. Methods for the economic evaluation of health care programmes. 3rd ed. Oxford: Oxford University Press, 2005:55-101.

13. Finkler SA. The distinction between cost and charges. Ann Intern Med 1982;96:102-9.

14. Suver JD, Cooper JC. Principles and methods of managerial cost-accounting systems. Am J Hosp Phar 1988;45:146-52.

15. Mishra L, editor. Drug today; October-December 2004. Delhi: Larina Publications (India) Inc., 2004.

16. Canadian Coordinating Office for Health Technology Assessment. Guideline for economic evaluation of pharmaceuticals: Canada. 2nd ed. Ottawa: CCOHTA Publications, 1997:39-47.

17. Edejer TT-T, Baltussen R, Adam T, Hutubessy R, Acharya A, Evans DB et al. Making choices in health: WHO guide to cost-effectiveness analysis. Geneva: World Health Organization, 2003:197-216.

18. Labour Bureau, Ministry of Labour and Employment, Chandigarh, India. (http://labourbureau.gov. in/CPI IW 2004, accessed on 22 October 2008). 